\title{
Figure(s) et mise en crise du discours philosophique : l'exemple de l'hypozeuxe dans Corpus de Jean-Luc Nancy
}

Figure(s) and crisis of the philosophical discourse: the example of the hypozeuxis in Corpus of Jean-Luc Nancy

\section{Mathilde Vallespir}

\section{(2) OpenEdition}

\section{Journals}

Édition électronique

URL : http://journals.openedition.org/pratiques/2404

DOI : 10.4000/pratiques.2404

ISSN : 2425-2042

Éditeur

Centre de recherche sur les médiations (CREM)

Référence électronique

Mathilde Vallespir, « Figure(s) et mise en crise du discours philosophique : I'exemple de l'hypozeuxe dans Corpus de Jean-Luc Nancy », Pratiques [En ligne], 165-166 | 2015, mis en ligne le 01 octobre 2015, consulté le 04 mai 2019. URL : http://journals.openedition.org/pratiques/2404; DOI : 10.4000/ pratiques. 2404

Ce document a été généré automatiquement le 4 mai 2019.

(c) Tous droits réservés 


\section{Figure(s) et mise en crise du discours philosophique : l'exemple de l'hypozeuxe dans Corpus de Jean-Luc Nancy}

Figure(s) and crisis of the philosophical discourse: the example of the hypozeuxis in Corpus of Jean-Luc Nancy

\section{Mathilde Vallespir}

Discours philosophique et rhétorique ne font pas bon ménage. Bien qu'en pratique aucun discours ne soit dénué de rhétoricité ${ }^{1}$, en théorie, la philosophie tient à distance la rhétorique. En effet, la vocation de la philosophie est de dire l'être ; elle est onto-logie ; le langage auquel elle recourt se doit d'être ce langage de l'être, langage de la vérité et, en conséquence, exclut toute rhétorique. Ainsi N. Charbonnel (1999 : 32-61, 36-37) rappelle-telle que les « courants dominants » de la philosophie tentent d'évincer les figures, et cite pour exemple J. Locke qui, dans l'Essai sur l'entendement humain de 1690 «demande », au nom de la vérité, "qu'on regarde les termes figurés comme une imperfection et un véritable abus de langage ». Ce langage «transparent » à l'idée, tel qu'il est fantasmé par la philosophie, repose principalement sur deux ressorts : un lexique univoque et une syntaxe logique. C'est à cette dernière que je m'intéresserai aujourd'hui. En effet, cette syntaxe du logos philosophique répond depuis Platon, comme le souligne M. Meyer (1986 : 10), à un modèle: celui de la proposition. C'est cette proposition logique (entendue comme prédication dont le prototype est $S$ est $P$ ) qui, par sa clôture, permet d'accéder à la vérité univoque de ce qui est. Or, ce modèle propositionnel parait inébranlable : en effet, pas même l'ample crise de la raison que connut l'Occident au tournant du $\mathrm{xx}^{\mathrm{e}}$ siècle ne fut capable de le remettre en question. C'est en tout cas le point de vue de M. Meyer qui, renvoyant dos à dos M. Heidegger, L. Wittgenstein, J.-P. Sartre et J. Derrida, développe l'analyse selon laquelle « l'empire [...] du modèle propositionnel se perpétue ainsi dans sa dénégation même » (ibid. : 129-130). 
2 L'œuvre de J.-L. Nancy, Corpus, me parait témoigner de la crise de la raison que l'on vient d'évoquer et incarner cette dernière : elle répond en effet à ce double mouvement que M. Meyer prête à cette philosophie de crise, recourant au modèle propositionnel tout en tentant de s'en départir. Au sein de ce travail dialectique opéré sur la langue dans Corpus, l'hypozeuxe a un rôle central à jouer. L'objet de cet article est ainsi de mettre en évidence le fait que cette figure constitue un des opérateurs par lesquels la relation entre logos philosophique et rhétorique se redéfinit, ou plus précisément, de décrire la façon dont cette figure affecte le logos philosophique, et par là le reconfigure.

\section{L'hypozeuxe dans Corpus}

3 Corpus, qui date de 1992 pour sa première version ${ }^{2}$, est tout arc-bouté sur la phrase rituelle qui, selon J.-L. Nancy, fonde notre culture occidentale ${ }^{3}$ : hoc est enim corpus meum. Le livre constitue un vaste commentaire de cette phrase. Son titre latin, au-delà de son aspect strictement citationnel, révèle d'emblée la nature méta-discursive de l'ouvrage.

En effet, l'objet du livre est de mesurer l'atavisme conceptuel pesant sur la conception du corps en Occident, ou pour le dire en d'autres termes, de déconstruire la tradition occidentale chrétienne et en proposer, en même temps, une voie de sortie, par une redéfinition de l'écriture du corps. Le texte de Corpus, comme presque tous ceux de J.L. Nancy, repose sur un réseau figural dense créant des relations et une dynamique logique complexe, à plusieurs niveaux, qui fait appel à différents types de figures, parmi lesquelles des figures lexicales, notamment figure étymologique, mais aussi paronomases ${ }^{4}$ , tropes (syllepses et métaphores en particulier), toutes ces figures étant agencées avec des figures syntaxiques. Au sein de cet agencement serré, l'hypozeuxe tient une place de choix.

\subsection{Définition(s) de l'hypozeuxe}

5 Il faudrait ici opérer un travail archéologique sur cette figure inégalement représentée dans les différents traités : absente chez P. Fontanier, elle se trouve mentionnée comme autre nom possible du parallélisme dans le Gradus (Dupriez, 1983 : 322), et est reversée parfois dans les catégories de figures de construction ou «de juxtaposition » que sont accumulation, épitrochasme, énumération et apposition, comme c'est le cas dans le Dictionnaire des figures de N. Ricalens-Pourchot ${ }^{5}$ (2003: 166-167). Elle répond à des descriptions variables, qui supposent un fonctionnement discursif et des fonctions pragmatiques différents. Parmi ces descriptions, on choisira celle qu'en donne G. Molinié (1997: 169) qui, dans la perspective d'une rhétorique renouvelée, définit l'hypozeuxe comme une figure de construction reposant sur des «reprises et des parallélismes grammaticaux, de nature morphologique et fonctionnelle ». Il lui confère une "grande efficacité stylistique » (ibid.), sans aller plus avant dans la spécification. Je m'appuierai sur cette définition, en prenant donc pour critère de l'hypozeuxe le fait qu'elle suppose la prise en compte de deux aspects, morphologique et syntaxique ; plus exactement, que le parallélisme syntaxique, qui suppose identité fonctionnelle des éléments appareillés, s'appuie sur une identité morphologique qui peut prendre la forme d'une répétition? C'est cette possible répétition qui engendre d'ailleurs le recouvrement partiel de l'hypozeuxe avec des figures de répétition telles l'anaphore ou, plus précisément, l'épanaphore ${ }^{8}$. Pour identifier les hypozeuxes, je m'inscrirai dans la conception 
pragmatique que propose M. Bonhomme (2005: 31), dans laquelle les figures comptent parmi leurs critères constitutifs l'effet qu'elles produisent: en effet, "pour être totalement réalisées et fonctionnelles ", elles « ont besoin d'être construites au niveau de leur réception ». Elles seront de plus structurellement tenues pour des schèmes discursifs, de sorte que c'est leur saillance particulière qui permettra de les identifier comme telles.

\subsection{Configurations}

Corpus est saturé de ce type de structures, dont l'emploi échappe au système du fait de la variété importante des formes qu'elle prend. Si cette quantité d'occurrences décourage d'établir un relevé complet des hypozeuxes de l'œuvre, il est toutefois possible d'en dresser une typologie. Quatre paramètres sont à prendre en compte dans cette fin : tout d'abord, la nature morphosyntaxique du site (foyer) de la figure: on trouve essentiellement dans l'œuvre des groupes nominaux (nom déterminé, non déterminé), des groupes prépositionnels et des propositions. Puis, la nature quantitative du parallélisme : en effet, si le parallélisme comporte une limite basse, dans la mesure où il doit au moins compter deux éléments, il ne comporte pas de limite haute : la construction en parallèle est ainsi de droit infinie, l'hypozeuxe pouvant alors se radicaliser en énumération. Ensuite, c'est la fonction des éléments mis en parallèle qui doit être prise en compte. Cette dernière est propre à recouvrir un quatrième et dernier niveau d'analyse : la relation de ces éléments à la structure logique du texte. On s'interrogera donc ici sur le rôle que joue au sein de la structure propositionnelle le syntagme mis en parallèle : il peut s'agir du thème de la prédication, du prédicat, ou de toute la prédication, la figure n'étant nullement contrainte à cet égard. Toutes ces possibilités sont d'ailleurs représentées dans Corpus.

7 Sur l'ensemble des occurrences identifiées dans l'œuvre, deux tendances se dégagent. D'une part, une prédilection pour les hypozeuxes portant sur le prédicat, comme c'est le cas dans cet exemple (Corpus, p. 27): «Le corps est [le monde non impénétrable, [le monde qui n'est pas d'abord soumis à la compacité de l'espace ${ }^{9}$.» D'autre part, une tendance à l'énumération, c'est-à-dire à une hypozeuxe comportant un nombre élevé d'éléments mis en parallèle. On citera ainsi une hypozeuxe à quatre éléments : «Corps [ comme un bout d'os, [comme [[un caillou, [[un grave, [[un gravier qui tombe à pic» ( ibid. : 21).

8 La figure procède ici d'une double duplication, et se joue sur deux niveaux syntaxiques distincts : le groupe prépositionnel [comme X] puis, au sein du groupe prépositionnel, au niveau de la séquence de la préposition, la structure impliquant donc une anaphore de «comme» puis de l'article indéfini «un». Mais le texte comporte des hypozeuxes impliquant bien plus d'éléments :

«Hoc est enim : ce monde-ci, ci-gisant, avec [sa chlorophylle, [sa galaxie solaire, [ ses roches métamorphiques, [ses protons, [sa double hélice désoxyribonucléique, [ son nombre d'Avogadro, [sa dérive des continents, [ses dinosaures, [sa couche d'ozone, [les rayures de son zèbre, [sa bête humaine, [le nez de cléopâtre, [le nombre de pétales de la marguerite, [le spectre de l'arc-en-ciel, [la manière de Rubens, [la peau du serpent python, [la figure que fait André sur cette photo du 16 janvier, [ce brin d'herbe et cette vache qui le broute, et [la nuance de l'iris de l'œil de qui lit ce mot, ici et maintenant » (ibid. : 31) ?

9 L'hypozeuxe est si développée qu'il n'y a plus lieu ici de décompter les éléments qu'elle comporte. Nous reviendrons sur l'analyse de cette occurrence, mais cette bascule de 
l'hypozeuxe en énumération n'est pas exceptionnelle dans Corpus, où abonde ce type de structures ${ }^{10}$.

\subsection{Fonctions pragmatiques}

10 Au-delà de cette caractérisation formelle, on peut s'interroger sur les fonctions pragmatiques à attribuer à la figure. L'hypozeuxe semble ici être essentiellement dotée de deux fonctions : esthétique bien sûr, mais au-delà et fréquemment, "cognitive ${ }^{11}$ ». Elle joue alors le rôle traditionnellement attribué aux figures d'outil de structuration de l'argumentation (ibid.). C'est le cas pour l'occurrence suivante :

«[...] et cette limite est le corps, [non pas comme une pure et simple extériorité au sens, [non pas comme on ne sait quelle «matière » intacte, intouchable, [...], [non pas donc, pour finir [[comme le corps, mais bien [[comme LE CORPS DU SENS » ( Corpus, p. 24).

11 La reprise lexicale met en valeur le parallélisme syntaxique et fonctionnel des groupes prépositionnels, articulés par la triple itération de la négation, elle-même soulignée par le redoublement du terme négatif («non pas»). Cette hypozeuxe complexe sert ainsi de lit à une structure logique oppositive (articulée par l'association de l'outil négatif «non pas » à l'adverbe adversatif «mais ») ; le parallélisme à trois éléments de la protase permet de faire attendre et par là de mettre d'autant plus en valeur la chute logique, qui constitue l'apodose de la phrase.

Bien que fondamentale, cette fonction cognitive de l'hypozeuxe est loin d'être spécifique à son fonctionnement au sein de Corpus; et se cantonner dans ses limites supposerait d'affaiblir la portée de cette figure dans l'écriture de J.-L. Nancy. Il ne faudrait en effet pas négliger la situation discursive pragmatique qui est celle de ce texte et les attentes qu'elle implique. Corpus relève ainsi d'un type de discours, le discours philosophique, qui dispose des attentes autant qu'il se construit par rapport à elles. Parmi celles-ci, il en est une centrale: que le texte réponde à cette vocation ontologique évoquée en introduction comme le fait du texte philosophique, qu'il soit ainsi ce «langage de l'être » que ce dernier se fixe pour fin.

\section{2. hypozeuxe et prédication : altération de la proposition}

\subsection{Hypozeuxe et prédicat}

Corpus, dans une certaine mesure, répond à une telle attente. L'ambition onto-logique de ce discours est évidente, et les énoncés correspondant à la forme canonique d'un tel langage de l'être, et donc au modèle propositionnel précédemment évoqué, sont légion. C'est le cas par exemple de l'énoncé initial du livre (" hoc est enim corpus meum », Corpus, p.7), mais on en relèverait de nombreux autres exemples, tels "L'égoïté est une signification (nécessaire) d'ego ", "l'amour est le toucher de l'ouvert ", «l'aréalité est l' ens realissimum » et «la masse est l'épaisseur » (ibid. : 26-27, 28, 39, 82), ou, sous forme négative : « la réalité n'est pas à voir » ou « Un corpus n'est pas un discours" (ibid. : 41, 46). Or, cette structure propositionnelle, déjà per se infléchie, notamment par des structures recourant à la médiation du pronom démonstratifi ${ }^{12}$, dont on connait le pouvoir brouillant ${ }^{13}$, se trouve précisément affectée par l'hypozeuxe, et en particulier par les deux 
structures caractéristiques que j'ai évoquées tout à l'heure: l'hypozeuxe portant sur le prédicat et l'hypozeuxe énumérative, ces deux profils de figure concordant souvent.

Tout d'abord, l'hypozeuxe affectant le prédicat prend très souvent la forme de l'alternative niée, telle "le corps n'est ni signifiant, ni signifié », ou "Les corps de notre monde ne sont ni sains, ni malades" (ibid.: 24, 93). Le modèle propositionnel $S$ est $P$ devient donc ici Sn'est ni $X$, ni Y, dans le cas d'une hypozeuxe minimale, avec cette particularité que $X$ et $Y$, les deux prédicats niés, se trouvent entretenir une relation d'opposition, qui repose sur une relation lexicale d'antonymie entre ces termes. Parce qu'ils expriment ainsi une impossibilité logique, ces énoncés mettent en péril le principe logique fondamental de non-contradiction.

Au-delà, la structure de l'hypozeuxe énumérative me parait peut-être plus caractéristique encore de l'écriture de J.-L. Nancy. Au $S$ est $P$ de la logique formelle se substitue alors, via l'hypozeuxe, un $S$ est $A, B, C, D \ldots n$. Une telle configuration parait avoir un double effet. Tout d'abord, elle parait ici retourner, ou inverser, le fonctionnement structurel logique attendu de l'hypozeuxe : en effet, si le parallélisme, on l'a vu, a un pouvoir structurant (ce qui permet à la figure d'être dotée de la fonction cognitive évoquée supra), sa prolifération ne peut que perdre le lecteur. Loin de jouer son rôle de guide, repère ou jalon, il fonctionne alors au contraire comme opérateur de déstructuration. De plus, d'un point de vue logique, cette démultiplication du prédicat altère l'unité conceptuelle du sujet posé.

\subsection{Du logos au catalogue}

C'est ce que l'on peut mesurer en revenant à l'exemple radical cité plus haut ${ }^{14}$ :

« Hoc est enim : ce monde-ci, ci-gisant, avec [sa chlorophylle, [sa galaxie solaire, [ ses roches métamorphiques, [ses protons, [sa double hélice désoxyribonucléique, [ son nombre d'Avogadro, [sa dérive des continents, [ses dinosaures, [sa couche d'ozone, [les rayures de son zèbre, [sa bête humaine, [le nez de Cléopâtre, [le nombre de pétales de la marguerite, [le spectre de l'arc-en-ciel, [la manière de Rubens, [la peau du serpent python, [la figure que fait André sur cette photo du 16 janvier, [ce brin d'herbe et cette vache qui le broute, et [la nuance de l'iris de l'œil de qui lit ce mot, ici et maintenant?»(Corpus, p. 31)

17 Ici l'énumération repose sur une hypozeuxe dont le parallélisme syntaxique s'appuie sur la reprise morphosyntaxique du déterminant possessif ( «sa chlorophylle, sa galaxie, son nombre d'Avogadro » etc.) puis de l'article défini ${ }^{15}$. Certes, comme le rappelle M. Meyer, il est un lieu, au sein de la proposition logique, qui peut accueillir le multiple : le prédicat ${ }^{16}$. Pour autant, cette multiplicité a des conditions : que le principe de non-contradiction soit respecté entre les prédicats, et qu'une cohérence suffisante régisse leur juxtaposition. Ceci suppose, d'un point de vue linguistique, une compatibilité sémantique entre les lexies jouant le rôle de prédicat. Si ces principes ne sont pas respectés, la multiplicité ne peut que remettre en cause l'unité conceptuelle du sujet qu'a précisément vocation à construire la proposition: elle fait ainsi obstacle à la fabrication même de cette unité. Dans l'exemple que l'on vient de donner, l'ensemble des prédicats se substitue au « corpus meum » de la formule consacrée. Ce parcours de prédicats opère ainsi un repli sur la deixis, comme le suggèrent les formes initiales de démonstratifs à valeur déictique («ce mondeci, ci-gisant »). Les éléments juxtaposés ensuite sont hétéroclites, et on peut s'interroger sur le principe propre à régir une telle hétérogénéité. Quelle serait ici la catégorie englobante, ou, en termes lexicaux, l'hyperonyme de l'ensemble? Même la catégorie la 
plus englobante qui soit, celle de l'être, parait insuffisamment étendue, comme le point d'interrogation final le laisse à penser. Ainsi, celui-ci, qui inscrit la phrase tout entière dans une modalité interrogative, suppose que soit suspendue la valeur de vérité de la proposition. La catégorie de l'être, loin d'être posée ici, se trouve donc à la fois grevée par la deixis et le multiple et maintenue dans un suspens hypo-thétique ${ }^{17}$. L'hypozeuxe énumérative met en œuvre dans l'écriture même du texte le changement de paradigme appelé de ses vœux par J.-L. Nancy quelques dizaines de pages plus loin : « Il faudrait un corpus, un catalogue au lieu d'un logos, l'énumération d'un logos empirique " (Corpus, p. 47). En se muant en dire du monde, l'hypozeuxe vient ainsi opérer cette mutation du logos en catalogue.

\section{Reconfigurer le logos philosophique : le Toucher de Jean-Luc Nancy}

18 Ainsi, dans Corpus, l'hypozeuxe a valeur exemplaire parmi les figures par la façon dont elle se configure dans la langue de ce texte et dont par là même elle reconfigure le type de discours dans lequel elle s'inscrit, le discours philosophique, en altérant le modèle propositionnel propre à une tradition philosophique issue de Platon. Dans sa polyfonctionnalité, au-delà d'autres possibles fonctions que la figure peut prendre, elle révèle ici tout particulièrement une fonction sémiotique, à la fois déconstructrice et reconstructrice. En effet, la figure rhétorique fonctionne ici contre le logos philosophique, elle le déconstruit en altérant son fondement logique, la propositionnalité. Au sein du langage, J.-L. Nancy fait ainsi travailler le rhétorique contre le logique. Mais cette destruction/déconstruction fonctionne comme le revers d'une reconstruction: car le philosophe fabrique la possibilité d'une nouvelle raison philosophique; il façonne une nouvelle langue philosophique qui configure par là une nouvelle pensée, qui est aussi une nouvelle logique. Cette langue a vocation de répondre à une poétique que le philosophe appelle, dans Corpus même, "le toucher "; puisque le corps ou corpus, comme le dit J ;L. Nancy, échappe au langage (« de là qu'il n'est pas possible d'écrire au corps, ou d'écrire le corps ", Corpus, p. 21), il faut fabriquer une langue capable de le «toucher ». Le travail de l'hypozeuxe dont j'ai essayé de rendre compte ici doit donc être réinscrit dans un travail rhétorique et linguistique plus large de la langue, dont la vocation est de reconfigurer le logos philosophique pour parvenir à ce « toucher ». Plus largement encore, une telle démarche (qui consiste en l'utilisation des pouvoirs rhétoriques de la langue contre la logique formelle, ce en toute conscience, en érigeant même cette démarche en principe d'écriture $\left.{ }^{18}\right)$, doit, je crois, être tenue pour symptomatique de l'écriture d'une certaine philosophie de la seconde moitié du $\mathrm{xx}^{\mathrm{e}}$ siècle ${ }^{19}$, et en particulier celle qui a été appelée " pensée $1968^{20}$ ». L'exemple de Corpus de Nancy permet de mesurer à quel point cette crise traversée par la raison en ce siècle est à entendre non comme une destruction mais comme une mutation propre à disposer les conditions de nouveaux modes de pensée. 


\section{BIBLIOGRAPHIE}

BonHomme, M. (2005) : Pragmatique des figures du discours, Paris, H. Champion.

CHARbonnel, N. (1999) : « Métaphore et philosophie moderne », in : N. Charbonnel, La Métaphore, entre philosophie et rhétorique, Paris, Presses universitaires de France, p. 32-61.

DUPRIEZ, B. (1983) : Gradus, les procédés littéraire : dictionnaire, Paris, Union générale d'éditions.

FERRY, L. \& RENAUT, A. (1988) : La Pensée 68 : essai sur l'anti-humanisme contemporain, Paris, Gallimard.

MEYER, M. (1986) : De la métaphysique à la rhétorique, Bruxelles, Éd. de l'Université de Bruxelles. - (2008) : Principia rhetorica. Une théorie générale de l'argumentation, Paris, Fayard.

NANCY, J.-L. (2006) [1992] : Corpus, Paris, Métaillié.

LE GOFFIC, P. (1993) : Grammaire de la phrase française, Paris, Hachette.

MOLINIÉ, G. (1997) : Dictionnaire de rhétorique, Paris, Le Livre de Poche.

MORIER, H. (1961) : Dictionnaire de poétique et de rhétorique, Paris, Presses universitaires de France.

RICALENS-POURCHOT, N. (2003) : Dictionnaire des figures de style, Paris, A. Colin.

VALLESPIR, M. (sous presse) : «Peut-on penser un style de la déconstruction ? La question du "style Derrida" ", in : É. Bordas (dir.), Style, langue, société, Bordeaux, Presses universitaires de Bordeaux. - (à paraitre) : « La métaphore dans Éperons de Derrida : figure et dé-figuration », intervention proposée dans le cadre de la journée d'étude Lire Derrida. Autour d'Éperons. Les styles de Nietzsche, organisée par D. Maingueneau et M. Vallespir, 8 février 2014, Université Paris Sorbonne.

\section{NOTES}

1. C'est là le point de vue le plus généralement défendu dans la linguistique actuelle, sortie aujourd'hui d'une pensée de la figuralité comme écart : voir M. Bonhomme (2005:27).

2. Ce travail prend pour référence l'édition revue et complétée de 2006.

3. Voir le début de Corpus (Nancy, 2006 : 7) : « Hoc est enim corpus meum : nous provenons d'une culture dans laquelle cette parole rituelle aura été prononcée, inlassablement, par des millions d'officiants de millions de cultes. Dans cette culture, tous la (re)connaissent, qu'ils soient ou non chrétiens. »

4. Voir par exemple J.-L. Nancy (2006: 86) : «Le corps créé est là, c'est-à-dire entre ici et là, abandonné [...] res, réel aréal, [...]. Corps seulement posés, pesés d'être seulement posés, et pesants, ouvrant, s'ouvrant leurs lieux». Le paragraphe, qui comporte initialement une amphibologie («entre » pouvant être tenu pour verbe ou pour préposition), est ici nettement marqué par la paronomase, qui se joue entre les participes « posés »/ "pesés » et avec l'adjectif verbal « pesants », mais aussi entre le latin et le français ( « res »/ « réel ») ; la figure parait même ici érigée en principe de progression discursive, comme c'est souvent le cas dans l'œuvre de J.L. Nancy. Pour la figure étymologique, voir J.-L. Nancy (ibid. : 29) : «L'ob-jection touche.» On peut lire également dans "touche " une syllepse, le verbe ayant ici à la fois son sens propre et 
figuré, la suite du texte entretenant cette ambigüité : «Ce corps, ce trait, cette zone de ce corps me touche (touche "mon" corps). Ça me plaît ou ça me déplaît, ça me contrarie ou non, ça m'intrigue ou non, ça me frappe ou ça me laisse indifférent, ça m'excite ou ça me révulse. »

5. Les frontières entre ces figures demanderaient peut-être à être précisées : ainsi, l'énumération et l'accumulation sont toutes deux définies comme « liste de mots de même nature et de même fonction "; elles s'opposent toutefois par le fait que "ces mots n'appartiennent pas à un même ensemble " pour la première, quand c'est au contraire le cas pour la seconde. Si une telle opposition parait elle-même contestable, du fait de la faible définition de la notion d'ensemble (dont on comprend qu'elle est sémantique), elle l'est plus encore au vu des exemples donnés : entre «[...] et là se fait entendre un perpétuel piétinement, caquètement, mugissement, beuglement, bêlement, meuglement, grondement, rognonnement, mâchonnement, broutement des moutons et des porcs et des vaches à démarche pesante » (p. 167, exemple emprunté à Joyce, cité par ailleurs par B. Dupriez, ibid.: 21) et «Je veux aller quérir la justice et faire donner la question à toute ma maison : à servantes, à valets, à fils, à filles et à moi-même ", la différence parait mince, seul le domaine sémantique d'emprunt des lexies étant un peu plus étroit dans le second exemple que dans le premier. Mais plus instable encore parait le critère d'identification de l'épitrochasme et sa distinction avec les précédentes figures, comme sa définition permet de le mesurer: "accumulation de mots ou syntagmes expressifs, souvent assez brefs. Ex: Don Fernand, dans sa province, est oisif, ignorant, médisant, querelleur, fourbe, intempérant, impertinent » (Ricalens-Pourchot, 2003 : 167). Si l'exemple recourt à une autre catégorie que le nom, celle de l'adjectif, la structure répond à la définition des deux précédentes figures, tous les éléments accumulés répondant à une nature et une fonction identiques, l'expressivité particulière de la série n'étant pas véritablement perceptible, encore moins propre à constituer un critère de spécification de la figure.

6. On opposera à ce point de vue sur la figure celui d'H. Morier (1961:548-549) qui en fait une figure d'ajout: "figure par laquelle on adjoint, à chacun des termes d'une énumération, des éléments seconds (verbe, adverbe, épithète, complément, proposition), de manière à maintenir un parallélisme dans la construction de la phrase." Il cantonne l'hypozeuxe dans un rôle esthétique, la figure ayant pour vocation de « rétablir l'équilibre » de la phrase, en s'en référant à la théorie dix-huitiémiste des figures : «En France, les théoriciens du XVIII ${ }^{\mathrm{e}}$ siècle se sont plus d'une fois préoccupés de ces problèmes, avec l'assentiment de l'Académie française ; il ne faut pas oublier, en effet, que l'Académie est plutôt formée d'écrivains que de linguistes ; et comme ils sont chargés d'établir les normes de la pure langue française, il s'ensuit que leurs soucis d'écrivains et d'esthètes de la langue se sont infiltrés dans la grammaire sous forme de préceptes où l'euphonie, l'eurythmie et l'expressivité jouent un rôle indéniable. »

7. Voir l'exemple choisi par G. Molinié (ibid) : « Du feu, j'ai tisonné les braises. De l'eau, j'ai retiré les spaghettis. D'elle, je me suis occupé, comme d'une princesse dans son palais, à croire qu'il en allait de ma tête. "

L'hypozeuxe est ici fondée sur la reprise du groupe prépositionnel, impliquant la répétition de la préposition «de » sous des morphologies différentes selon qu'elle apparait telle quelle («de l'eau »), qu'elle est amalgamée à l'article défini masculin (« du»), ou qu'elle subit une élision devant voyelle (« d'elle »).

8. On remarquera que l'exemple donné par G. Molinié ne peut être véritablement rabattu sur une anaphore ou épanonaphore, du fait de la variation morphologique de la préposition.

9. On note, dans cet exemple et les suivants, le début des syntagmes mis en parallèle par un crochet, simple ou double selon le niveau syntaxique, et en gras, les éléments du syntagme qui sont répétés.

10. Voir par exemple (Corpus, p. 93) : «Les corps écotechniques sont un autre genre de créatures, pressées de toutes parts, de toutes masses en elles-mêmes, à travers elles et entre elles, branchées, échographiées, radiographiées, les unes à travers les autres, communiquant leurs 
résonances nucléaires, contrôlant leurs déficits, s'ajustant sur leurs défaillances, appareillant leurs handicaps, leurs trisomies, leurs muscles fondus, leurs synapses effondrées, de toutes parts accolées, collées, mêlées, infiltrées par milliards de corps dont pas un seul ne tient en équilibre sur un corps, tous glissants, ouverts, répandus, greffés, échangés. », ou p. 87 : «Corpus de pesée d'une matière, de sa masse, de sa pulpe, de son grain, de sa béance, de son môle, de sa molécule, de sa tourbe, de son trouble, de sa turgescence, de sa fibre, de son jus, de son invagination, de son volume, de sa pointe, de sa tombée, de sa viande, de sa concrétion, de sa pâte, de sa cristallinité, de sa crispation, de son spasme, de sa fumée, de son nœud, de son dénouement, de son tissu, de sa demeure, de son désordre, de sa blessure, de sa douleur de sa promiscuité, de son odeur, de son jour, de son goût, de son timbre, de sa résolution, de son haut et bas, droite et gauche, de son acidité, de son essoufflement, de son balancement, de sa dissociation, de sa résolution, de sa raison... ", mais aussi, entre autres, p. 89, 91, 100.

11. M. Bonhomme, op. cit., p. 172, prête cette fonction à la répétition anaphorique, sur laquelle s'appuie l'hypozeuxe, et qui a pour vocation la « mise en relief du message transmis ».

12. C'est le cas pour la dislocation avec reprise anaphorique opérée par le pronom démonstratif, du type $X$, c'est $Y$ comme c'est le cas dans Corpus (p. 24): "Le corps du sens n'est en rien l'incarnation de l'idéalité du "sens" : c'est au contraire la fin de cette idéalité, la fin du sens ».

13. Voir P. Le Goffic (1993 : 144) qui évoque « l'effet [...] de déplacement, d'écran », produit quand l'anaphore d'un nom est opérée par le démonstratif.

14. Voir 1.2.

15. J'ai indiqué en gras les déterminants possessifs et en italiques les articles définis.

16. C'est là d'ailleurs ce qui oppose la logique platonicienne et aristotélicienne : voir M. Meyer (2008 : 28) : «L'être est un comme sujet et multiple comme prédicat : la codification de la structure de la proposition est née. Tout énoncé aura nécessairement la forme $S$ est $P$. »

17. On trouve, un peu auparavant dans le texte, une autre "solution » rhétorique pour rendre compte de cette ouverture non totalisable, sous la forme d'une énumération ouverte sur une aposiopèse : « Autrui sera d'abord venu, du plus loin, du plus écarté, dans un corpus de traits qui finit par s'identifier à “ lui" - et qui pourtant reste en lui-même inidentifiable : car ces traits sont tous étrangers les uns aux autres, ce bras avec ce menton, ces poils avec ces hanches, et cette voix, et cet................tous faisant corps et disloqués ensemble.»(Corpus, p. 30). Les points de suspension, forme graphique de l'aposiopèse, manifestent ainsi l'impossibilité de circonscrire Autrui par le langage.

18. Ce travail de la langue, pour trouver le corps au-delà du logos mais dans le langage, est présenté par exemple dans Corpus (p. 20) : "(Si j'écris, je fais des effets de sens - je place tête, ventre et queue - et je m'écarte donc des corps. Mais justement: il faut ça, il faut une mesure infinie, toujours retracée de cet écart. L'excription passe par l'écriture - et certainement pas par des extases de la chair ou du sens. Il faut donc écrire, depuis ce corps que nous n'avons pas, et que nous ne sommes pas non plus : mais où l'être est excrit. [...]) » Le langage du corps est donc à trouver dans cet écart du corps à lui-même que suppose «l'excription ». Celle-ci implique à son tour une écriture envisagée comme lieu de travail du logos par le rhétorique, dont nous j'ai mis en évidence un aspect dans cet article.

19. Voir également à cet égard $M$. Vallespir (sous presse, à paraitre).

20. Par ses détracteurs en particulier : voir L. Ferry \& A. Renaut (1988). 


\section{RÉSUMÉS}

On envisage ici la figure dans le cadre d'un type de discours qui a priori s'en méfie, voire l'exclut : le discours philosophique. À la lumière d'un exemple, celui de l'hypozeuxe dans Corpus de JeanLuc Nancy, on mettra en évidence le fait qu'au sein d'un réseau figural complexe, cette figure opère une dé- et une reconfiguration discursive : on montre que, loin de servir d'ornement, loin, plus encore, de servir l'organisation logique du discours, elle porte atteinte à la clé de voute de ce discours, en affectant son fonctionnement logique, et plus précisément prédicatif. Après avoir décrit le mécanisme dé/reconfigurant de la figure, nous mettons en valeur dans quelle mesure un tel fonctionnement figural, qui redéfinit contextuellement la valeur de la figure, est propre à fabriquer un mode inédit de régime logique philosophique, et témoigne par là d'une mise en crise de la raison philosophique dans le dernier quart du $\mathrm{xx}^{\mathrm{e}}$ siècle en France.

The hypozeuxis is here considered in a context that, at first sight, is wary of it, or even expels it: the philosophical discourse. The hypozeuxis will deal as an example to show that in Corpus of J.L. Nancy, among many other rhetorical figures, this one happens to be an operator of a renewing of the philosophical discourse. Indeed, far from being just a discourse ornament or farther, a tool of logical organisation of the discourse, the hypozeuxis is affecting the ground of this discourse: his logical and predicative organisation. After having shown the way the figure works as an operator of renewing of the logical structures of the language, we show how this hypozeuxis'way of working, which redefines his rhetorical value, is building a new way of philosophizing, and attests to the crisis of philosophical reason that happened in the last quarter of the $20^{\text {th }}$ century.

\section{INDEX}

Mots-clés : rhétorique et philosophie, rhétorique et logique, crise de la raison, écriture philosophique, Jean-Luc Nancy, hypozeuxe

Keywords : rhetoric and philosophy, rhetoric and formal logic, crisis of reason, philosophical writing, hypozeuxis

\section{AUTEUR}

\section{MATHILDE VALLESPIR}

Stih (EA 4609), Université Paris Sorbonne-Paris 4 